\title{
Serum cystyl aminopeptidase activity in the 36th week of pregnancy
}

\author{
B. H. DURHAM AND R. E. REWELL \\ From Liverpool Maternity Hospital, UK
}

SUMMARY Serum cystyl aminopeptidase (CAS) activity was estimated at 36 weeks' gestation in 209 normotensive pregnancies. The highest activity was found in 31 women who had spontaneous deliveries before 38 weeks' gestation and the lowest in 76 women who were induced after term. The enzyme levels in 117 women who developed hypertension of pregnancy were higher than for normotensives; the highest levels were found in 32 women with pre-eclampsia. A correlation was found between serum CAS activity at 36 weeks' gestation and the birth weight of babies of women who went into spontaneous labour at term (277 to 283 days' gestation).

Cystyl aminopeptidase (CAS, oxytocinase, $a$ aminoacyl-peptide hydrolase, EC 3.4.11.3) is synthesised in the placenta and appears in the maternal circulation. Its estimation in serum has been used to assess placental function by several investigators (Chapman et al., 1971; Hurry et al., 1972; Petrucco et al., 1973). All found a wide range in the activity of the enzyme in serum near term.

CAS destroys oxytocin, and Klimek (1967) postulated an oxytocinase/oxytocin balance system to control the level of the hormone and protect the uterus from its action when the level rises near term; as long as the placenta was functioning normally the concentration of the enzyme would in some way mirror the concentration of circulating oxytocin. This might explain the wide range of CAS activity encountered in the third trimester of pregnancy.

The present investigation was undertaken to determine whether or not there is any significant difference in the serum CAS levels of women in whom labour occurs spontaneously at or before term (defined as 277 to 283 days' gestation) and those whose labour is delayed (284 days' gestation or later). Calculation of the duration of gestation still depends on knowing the first day of the woman's last menstrual period, and this is not always easy to determine. As laboratory workers we had to rely on the judgement of our clinical colleagues. As obstetricians they must determine a date on which to base important decisions regarding management of the patient. We rejected for study patients in

Received for publication 12 June 1978 whom the obstetricians were unable to make a decision on this point.

\section{Methods}

Enzyme activity at 36 weeks of pregnancy was estimated in women chosen at random, and subsequently they were divided into two categories: those in whom labour had occurred spontaneously and those in whom it was induced at or after 284 days' gestation. Only normotensive patients were included: they were excluded from this group if they developed any signs of hypertension during the remainder of their pregnancy. The criteria for hypertension during pregnancy accepted by the clinicians with whom we work is a blood pressure reading of at least $140 / 90 \mathrm{mmHg}$, which is confirmed after more than 24 hours' rest in hospital. Furthermore, a rise of $10 \mathrm{mmHg}$ in diastolic pressure over that obtained at a previous visit is regarded with suspicion, and the patient is treated as hypertensive if this is sustained after rest. For the diagnosis of pre-eclampsia, proteinuria is demanded in addition to hypertension.

A group of hypertensive patients was chosen and studied in the same way as the normotensives.

A blood sample was collected from each of 326 women during the 36th week of pregnancy into a vial containing no anticoagulant, and the serum, after separation, was stored at $-20^{\circ} \mathrm{C}$ until assayed for CAS activity. At this temperature the enzyme activity remains stable for at least six months (Durham, 1974, unpublished). CAS activity was assayed by a method which has s-benzyl-L-cysteine4-nitroanilide as the substrate (Durham, 1976). 
Table Mean CAS activity and birth weight in normotensive, hypertensive, and pre-eclamptic pregnancies

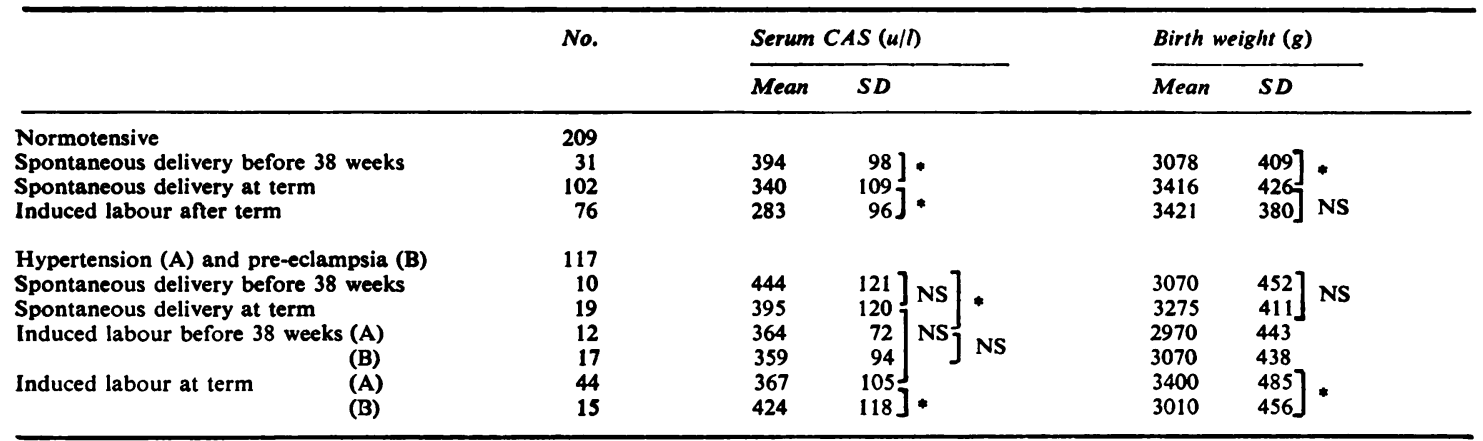

NS $=$ not significant $(P>0.05) ;{ }^{*} P<0.01$

In the earlier stages, nearly all the women who came to the antenatal clinics were admitted to the study. Later, only hypertensives were selected to provide enough patients for statistical analysis. Where the development of erythroblastosis seemed possible the patient was excluded. All the babies survived. We recorded the time at which delivery took place, whether or not the onset of labour was spontaneous, the birth weight of the baby, and its sex. The mean and standard deviation of the CAS activity and the birth weight of the babies was calculated for each defined group (Table). The differences found were examined for statistical significance using Student's $t$ tests.

\section{Results (Table)}

We found no significant difference between serum CAS levels of women who gave birth to boys and those who had girls. Some of the patients who were induced might well have delivered spontaneously within a few days if they had been left, so creating a bias in the results from the induced groups.

In normotensive women, the highest CAS levels were found when the onset of labour occurred spontaneously before 38 weeks. A significant difference $(P<0.05)$ was found between these women and those who delivered spontaneously at term and also between this latter group and those who were induced at term. With regard to birth weights, no significant difference was found between the two groups delivering at term, but the difference between the groups delivering spontaneously was significant. This would be expected if the date of the last menstrual period for the spontaneous group was correct.

In women with hypertension, the highest CAS levels were also found in the group that went into early spontaneous labour. A significant difference was found between these patients and those who were induced at term. But, unlike the normotensives, the difference between the two spontaneous groups was not significant. This may be explained by the small numbers in each group.

When the patients with hypertension and those with pre-eclampsia were compared, a significant difference was found between those who were induced at term, but no significant difference was found between those in these two classes who were induced before term.

All patients with hypertension had higher CAS levels than the corresponding normotensives; the difference between those who delivered spontaneously at term was significant. However, there was no significant difference between these two groups if only patients who went into labour spontaneously before 38 weeks' gestation were considered $(P=0 \cdot 1)$.

The association between CAS activity and birth weight was investigated for women delivered at or after term, for both normotensives and hypertensives. Women with pre-eclampsia were not considered for this. From Figs 1 and 2 it will be seen that there is some correlation between CAS levels and birth weight. The significance of the correlation for normotensives was at the $5 \%$ level, but it was at the $10 \%$ level for hypertensives.

\section{Discussion}

Klimek (1967) suggested that synthesis of CAS is induced by oxytocin. This might explain our finding that in normotensive mothers the highest CAS levels were found in those who delivered spontaneously before 38 weeks' gestation and the lowest were in those induced after term.

Variation in oxytocin production would also explain, on this hypothesis, the wide range of CAS levels found during the third trimester. However, 


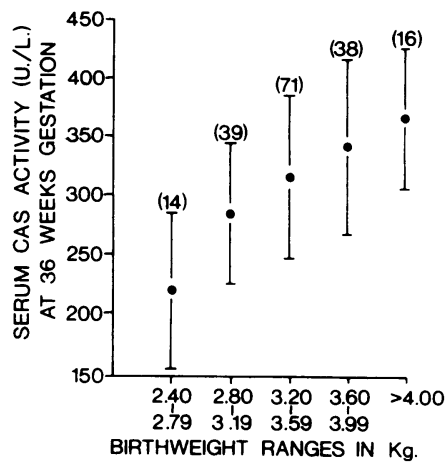

Fig. 1 Relation between serum CAS activity at 36 weeks' gestation and birth weight in normotensive term pregnancies. Mean and standard deviation given for each range. The figures in parentheses are the number of pregnancies in each range.

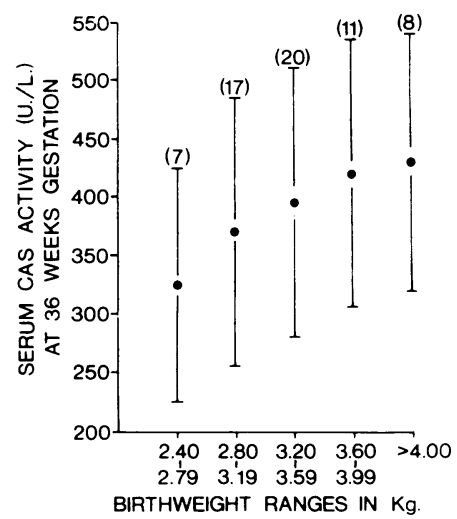

Fig. 2 Relation between serum CAS activity at 36 weeks' gestation and birth weight in hypertensive pregnancies that went to term. Mean and standard deviation given for each range. The figures in parentheses are the number of pregnancies in each range.

mothers with hypertension all had higher serum CAS levels than normotensive mothers, suggesting an additional stimulant to the synthesis of CAS in hypertension. The nature of this stimulant is unknown, but it may be noted that vasopressin can be degraded by CAS (Sjöholm and Yman, 1967), as can oxytocin.

Serum CAS levels could be used as a rough guide to birth weight in normotensive mothers but not in mothers with hypertension or pre-eclampsia, which are associated with high CAS levels, although the babies tend to be of low birth weight. Hashimoto (1961) made serial assays of CAS and found that a greater rate of increase was associated with preterm delivery, and maximum and minimum limits for weekly CAS increases have also been laid down (Chapman et al., 1973). By using these, it might be possible to exclude anticipated premature spontaneous deliveries when foretelling the birth weight.

Serial measurement of CAS activity may thus be used to monitor placental function and to predict preterm labour and also birth weight in normotensive patients only.

\section{References}

Chapman, L., Burrows-Peakin, R., Jowett, T. P., Rege, V. P., and Silk, E. (1973). The normal serum cystine aminopeptidase (CAP) range in pregnancy. Clinica Chimica Acta, 47, 89-92.

Chapman, L., Silk, E., Skupny, A., Tooth, E. A., and Barnes, A. (1971). Spectrofluorimetric assay of serum cystine aminopeptidase in normal and diabetic pregnancy compared with total oestrogen excretion. Journal of Obstetrics and Gynaecology of the British Commonwealth, 78, 435-443.

Durham, B. H. (1976). Spectrophotometric end-point method for assay of serum cystyl-aminopepsidase in pregnancy. Clinical Chemistry, 22, 79-82.

Hashimoto, T. (1961). Studies on L-cystine-aminopepsidase. Journal of the Japanese Obstetrical and Gynaecological Society, 8, 87-104.

Hurry, D. J., Tovey, J. E., Robinson, D. A., Beynon, C. L., and Cooper, K. (1972). Cystine aminopepsidase in normal and complicated pregnancies. Journal of Obstetrics and Gynaecology of the British Commonwealth, 79, 788-793.

Klimek, R. (1967). Relative duration of human pregnancy and oxytocin therapy. Gynaecologica (Basel), 163, 48-60.

Petrucco, O. M., Cellier, K., and Fishtall, A. (1973). Diagnosis of intra-uterine fetal growth retardation by serial serum oxytocinase, urinary oestrogen and serum heat-stable alkaline phosphatase (HSAP) in uncomplicated and hypertensive pregnancies. Journal of $\mathrm{Ob}$ stetrics and Gynaecology of the British Commonwealth, 80, 499-505.

Sjöholm, I., and Yman, L. (1967). Degradation of oxytocin, lysine vasopressin, angiotensin II and angiotensin-II-amide by oxytocinase (cystine aminopepsidase). Acta Pharmaceutica Suecica, 4, 65-76.

Requests for reprints to: Dr R. E. Rewell, Department of Pathology, Royal Liverpool Children's Hospital, City Branch, Myrtle Street, Liverpool L7 7DG, UK. 